\title{
Analysis of complex traits: mutagenesis versus QTLs
}

\begin{abstract}
I read with great interest the Commentary ${ }^{1}$ 1 comparing mutagenesis and the dissection of quantitative trait loci (QTL). It reminded me of past genetic controver$\operatorname{sies}^{2-7}$. Although I agree that the QTL strategy warrants criticism, in part to motivate efforts to overcome its difficulties, a balanced overview of the two strategies would have been more helpful in advancing quantitative genetic research.

Expression of many common diseases (such as hypertension, diabetes, alcoholism and so on) is influenced by both 'invariant' genes and 'variant' genes. The ability of the QTL strategy to find variant genes is a major advantage that justifies its
\end{abstract}

use. QTL strategies can use various tools including phenotypic selection ${ }^{8,9}$ to focus directly on sets of naturally occurring variant genes and their interactions.

Mutagenesis induces variants, which include both the invariant genes and variant genes of complex traits. Considering the function of a highly complex system, mutagenesis can create perturbations in a subsystem by induced-mutation, which occurs with an extremely low probability in the natural variation of that subsystem, but it can have a major effect on another subsystem, therefore affecting the expression of the complex phenotype assayed. Although such an induced variant may be

interesting to study what is possible in a system, it seems irrelevant in understanding the pathways of the naturally occurring expression of the phenotype. An induced mutation may give rise to a simalcrum of the phenotype, but we cannot rely on mutagenesis to deliver the genetic variants underlying many common diseases.

\section{Csaba Vadasz}

Laboratory of Neurobehavioral Genetics, New York University Medical Center, Nathan Kline Institute for Psychiatric Research,

Orangeburg, New York 10962, USA (e-mail: vadasz@nki.rfmh.org)

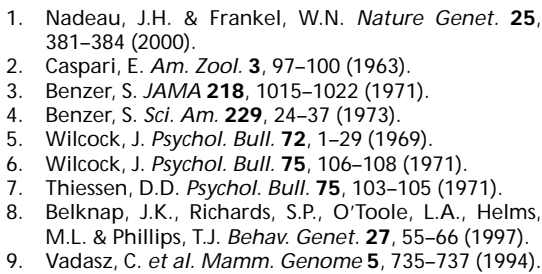

\section{Genetic homogeneity of Icelanders}

Several characteristics make populations suitable for gene mapping, including well-documented and universally accessible healthcare and a high cooperation rate. Moreover, extensive genealogy provides greater control over the search for identity by descent, both phenotypically and genetically. Another factor recently debated on these pages is the merit of homogeneous versus heterogeneous populations. Many biological measures of diversity have been applied to identify suitable populations, including heterozygosity of autosomal markers, mitochondrial sequence diversity and linkage disequilibrium (a favourite of those promoting genome-wide association studies, which are not currently feasible because they are far too expensive). Key to mapping studies is that such measures reflect the probability that two sequences are identical by descent. Árnason and colleagues ${ }^{1}$ challenge the notion that Ice-

\begin{tabular}{lc}
\hline \multicolumn{1}{c}{ Table $1 \bullet$ Genetic variation in Europe } \\
\hline & Heterozygosity \\
Germany & 0.443 \\
Norway & 0.441 \\
England & 0.437 \\
Denmark & 0.435 \\
Holland & 0.434 \\
Scotland & 0.432 \\
Faroes & 0.431 \\
Ireland & 0.428 \\
Isle of Lewis & 0.426 \\
Iceland & 0.424 \\
\hline
\end{tabular}

Data are available at $h t t p: / / w w w . d e c o d e . i s$ landers are a relatively homogeneous population, implying that this is a fiction promoted by a biotechnology company based in Iceland.

First, they interpret heterozygosity values of 11 classical genetic markers ${ }^{2}$ as demonstrating that Icelanders are no more homogeneous than other European populations. Our analysis of a larger data set of allele frequencies from 14 classical markers ${ }^{3,4}$ reveals that Icelanders have the lowest average heterozygosity of 10 European populations (Table 1 ).

Second, Árnason et al. ${ }^{1}$ dispute Icelandic homogeneity by comparing our published ${ }^{5}$ average heterozygosity for 298 Genethon markers used in linkage studies $(0.75$ for Icelanders and 0.79 for French $\mathrm{CEPH}$ individuals) with the average heterozygosity of all 5,264 Genethon markers (0.70) in the French population ${ }^{6}$. As a rule, comparisons of heterozygosity values are not valid unless based on the same set of loci in each population. This particular comparison is misleading, however, as the 298 markers were specifically selected for gene mapping on the basis of high heterozygosity. This subset of loci indicates Icelandic relative homogeneity, a finding supported by average heterozygosity of 100 SNP markers typed in our laboratory, which was 0.25 in Iceland compared with 0.28 in CEPH individuals.

Finally, they report a high nucleotide diversity in 73 new Icelandic mitochondrial sequences, claiming that Icelanders are "among the most variable Europeans" at this locus. They correctly state that this finding was previously reported in our study ${ }^{4}$ of the entire mitochondrial control region from 401 Icelanders. We disagree with their interpretation, and posit that high nucleotide diversity merely demonstrates that the mitochondrial sequences carried by female ancestors of the Icelandic contemporary population differed by a relatively large number of bases. Its relation to the number or geographic mixture of female founders is unclear.

The probability that two randomly selected sequences are identical by descent should be higher in populations with relatively few ancestors. Our previous study ${ }^{7}$ revealed a comparatively small effective population size of females in Iceland (based on low numbers of segregating sites and mitochondrial haplotypes)—only slightly larger than those estimated for other isolates such as Basques, Finns and Saami. This indicates that the Icelanders have relatively few ancestors and supports earlier claims that the contemporary Icelandic gene pool is suitable for gene-mapping studies.

\section{Jeff Gulcher ${ }^{1}$, Agnar Helgason ${ }^{1,2} \&$ Kári} Stefánsson ${ }^{1}$

${ }^{1}$ DeCODE genetics, Lynghals 1, Reykjavik, Iceland. ${ }^{2}$ Institute of Biological Anthropology,

University of Oxford, Oxford, UK.

1. Arnason, E., Sigurgislason, H. \& Benedikz, E. Nature Genet. 25, 373 (2000)

2. Bjarnason, O. et al. Ann. Hum. Genet. 36, 425-458 (1973).

3. Cavalli-Sforza, L.L., Menozzi, P. \& Piazza, A. The History and Geography of Human Genes (Princeton University Press, Princeton, 1994)

4. http://crick.stanford.edu/hg

5. Gulcher, J.R. et al. Nature Genet. 17, 84-87 (1997).

6. Dib, C. et al. Nature 380, 152-154 (1996).

7. Helgason, A. et al. Am. J. Hum. Genet. 66, 999-1016 (2000). 